\title{
Combined Local Global Optical Flow Approach in Bio-medical Image Sequences for blood flow detection
}

\author{
Kazi Md. Shahiduzzaman ${ }^{1}$, Khan Mamun Reza ${ }^{2}$, Nusrat Tazin ${ }^{3}$ \\ Lecturer, Electronics and Communication Engineering, JatiyaKabiKaziNazrul Islam University, \\ Mymensingh, Bangladesh ${ }^{1}$ \\ Assistant Professor, Electronics and Communication Engineering, JatiyaKabiKaziNazrul Islam University, \\ Mymensingh, Bangladesh ${ }^{2}$ \\ Lecturer, Electrical and Electronic Engineering, Northern University Bangladesh, Dhaka, Bangladesh ${ }^{3}$
}

\begin{abstract}
This paper addresses the optical flow analysis and the smoothing effect in Local and Global Differentiation. There are lots of Local and Global methods and their variants available. But in this paper, as a prototype of local methods least-square fit of Lucas and Kanade has been employed, while the Horn and Schunck approach has been considered as the representative for a global method. Each of these methods has their advantages and shortcomings. Which are mentioned in their respective section. As a consequence, the proposed made from the hybridization algorithm incorporates the advantages of both field and possess less disadvantages. Improvement is made in our algorithm using the spatio-temporal approach and appropriate differentiation approximation
\end{abstract}

Keywords: Optical flow, combined local global method, Spatio-temporal optical flow.

\section{INTRODUCTION}

In the clinical practice the motion detection process mainly relies on the expertise's visualization inspection or the manual measurement which is time consuming and at the same time it may vary from the expert to expert. The motion detection process is very important for the analysis of blood flow through the brain, the cardiac motion i.e. the myocardial motion in order to determine the abnormality by an effective means. The motion detection process helps the physician to their diagnoses process. Not only limited to medical analysis, motion detection also expands to stereo analysis, tracking and multimedia production. The effective and accurate determination of motion is a challenging topic in Computer Vision. B.D. Lucas T.Kanade published a paper named "An iterative registration technique with flow application to stereo" [1] which is known as the Local Method of Optical Flow. This method provides noise robustness. At the same time B.K.P. Horn and B.G. Schunk published their work named "Determining the Optical Flow" [2]. This method is know as Global method which provides 100 percent density in theoptical flow. There were also derivatives of these methods among the Black and Anandan's optical flow spatiotemporal approach was mentionable. Andreeset. al. published the paper "Lucas/Kanade meets Horn Schunk: Combining Local and Global Optic Flow Methods" [3] which is a noble approach to combine all the advantages of Local and Global methods. The most recent work about optical flow was done by Ahmad et. al. on their "CLG optical flow approach and frequency analysis of cranial ultrasonogram image Sequencce" [4]. In this paper they introduces CLG optical flow to the cranial ultrasonogram image sequence to determine the direction of blood flow through the brain. In this paper the optical flow analyzes was primary factoring but in addition smoothing effect in Local and Global Differentiation and Pre smoothing has also been analyzed. There are lots of Local and Global methods and their variants available. This Research addresses on Combined Local Global(CLG) approach, i.e., the least-square fit of Lucas and Kanade has been used as a prototype of local methods, while the Horn and Schunck approach has been used as a representative for a global method.

The rest of the paper is organized as follows. Section II describes the basics of combined local global method. The proposed algorithm for CLG is described in section III. Section IV illustrates the experimental results. Finally, section $\mathrm{V}$ concludes the paper.

\section{Combined local Global Method}

The optical flow is the pattern of apparent motion of objects, surfaces, and edges in a visual scene caused by the relative motion between an observer (an eye or a camera and the scene. Sequences of ordered images allow the estimation of motion as either instantaneous image velocities of discrete image displacements.

The Horn-Schunck method of estimating optical flow is a global method which introduces a global constraint of smoothness in the flow over the whole image. Thus, it tries to minimize distortions in flow and prefers solutions which show more smoothness. The flow is formulated as a global energy function which is then sought to be minimized: 


$$
\begin{gathered}
E=\iiint\left[\left(I_{x} u+I_{y} v+I_{t}\right)^{2}+\alpha^{2}\left(\left|\nabla_{u}\right|^{2}\right.\right. \\
\left.\left.+\left|\nabla_{v}\right|^{2}\right)\right] d x d y
\end{gathered}
$$

where $I_{x}, I_{y}$ and $I_{t}$ are the derivatives of the image intensity values along the $\mathrm{x}, \mathrm{y}$ and time dimensions respectively $v=[u, v]^{T}$, is the optical flow vector, and the parameter $\alpha(>0)$ is a regularization constant.

\section{A. Spatial Approach}

In order to design a combined local-global (CLG) method, the notations expressed Eqn. 2-5 are used [5].

$$
\begin{gathered}
w=(u, v, t)^{T} \\
\left(\left|\nabla_{w}\right|^{2}=\left|\nabla_{u}\right|^{2}+\left|\nabla_{v}\right|^{2}\right) \\
\nabla_{3} f=\left(f_{x}, f_{y}, f_{z}\right)^{T} \\
J \rho\left(\nabla_{3} f\right)=k \rho *\left(\nabla_{3} f \nabla_{3} f^{T}\right)
\end{gathered}
$$

It becomes evident that the Lucas-Kanade method minimizes the quadratic form illustrated in Eqn. 6

$$
J \rho\left(\nabla_{3} f\right)=k \rho *\left(\nabla_{3} f \nabla_{3} f^{T}\right)
$$

The Horn-Schunck technique minimizes the function shown in Eqn. 7.

$$
E_{H S}(w)=\int w^{T} J_{0}\left(\nabla_{3} f\right) w+\alpha\left(\left|\nabla_{w}\right|^{2}\right) d x d y
$$

This terminology suggests a natural way to extend the Horn-Schunck function to the desired CLG functional. After replacing the matrix $J_{0}\left(\nabla_{3} f\right)$ by the structure tensor $J_{\rho}\left(\nabla_{3} f\right)$ with some integration scale $\rho>0$, Eq. 8 can been found.

$$
\begin{aligned}
E_{C L G}(w)=\int_{\Omega} & \mid w^{T} J_{\rho}\left(\nabla_{3} f\right) w \\
& +\alpha\left(\left|\nabla_{w}\right|^{2}\right) \mid d x d y
\end{aligned}
$$

Its minimizing flow field (u, v) satisfies the EularLagrange equations represented in Eq. 9 and 10.

$$
\begin{gathered}
\begin{array}{c}
0=\Delta u-\frac{1}{\alpha}\left(K_{\rho} * f_{x}^{2} u+K_{\rho} * f_{x} f_{y} v+K_{\rho}\right. \\
\left.* f_{x} f_{t}\right)
\end{array} \\
0=\Delta v-\frac{1}{\alpha}\left(K_{\rho} * f_{y}^{2} v+K_{\rho} * f_{x} f_{y} u+K_{\rho}\right. \\
\left.* f_{x} f_{t}\right)
\end{gathered}
$$

\section{B. Spatiotemporal Approach}

The previous approaches used only spatial smoothness operators. Rapid advances in computer technology, however, make it now possible to consider also spatiotemporal smoothness operators. Formal extensions in this direction are straightforward. In general, one may expect that spatiotemporal formulations give better results than spatial ones because of the additional de-noising properties along the temporal direction. In the presence of temporal flow discontinuous smoothing along the time axis should only be used moderately. However, even in this case one can observe the beneficial effect of temporal information. A spatiotemporal variant of the LucasKanade approach simply replaces convolution with 2-D Gaussians by spatiotemporal convolution with 3-D Gaussians. These still leads to a 2 x 2 linear system of equations for the two unknowns' $u$ and $v$. combining the temporal extended variant of both the Lucas-Kanade and the Horn-Schunck method, spatiotemporal version of our CLG function like Eqn. 11 is found.

$$
\begin{array}{r}
E_{C L G}(w)=\int_{\Omega x[0, T]}\left[w^{T} J_{\rho}\left(\nabla_{3} f\right) w\right. \\
\left.+\alpha\left(\left|\nabla_{w}\right|^{2}\right)\right] d x d y
\end{array}
$$

Where convolutions with Gaussians are now to be understood in a spatiotemporal way and it is shown in Eqn.12.

$$
\left|\nabla_{3} w\right|^{2}=\left|\nabla_{3} u\right|^{2}+\left|\nabla_{3} v\right|^{2}
$$

Due to the different role of space and time the spatiotemporal Gaussians may have different standard derivations in both directions. If $J_{m n}$ is the component (n, $\mathrm{m})$ of the structure tensor, $J_{\rho}\left(\nabla_{3} f\right)$, the Eular-Lagrange equations are given by Eqn. 13 and Eqn.14.

$$
\begin{aligned}
& 0=\nabla_{3} u-\left(\frac{1}{\alpha} J_{11} u+J_{12} v+J_{13}\right) \\
& 0=\nabla_{3} v-\left(\frac{1}{\alpha} J_{21} u+J_{22} v+J_{23}\right)
\end{aligned}
$$

If the spatial Laplacean is replaced by the spatiotemporal Laplacian Eqn. 15 will be obtained.

$$
\Delta_{3}=\partial_{x x}+\partial_{y y}+\partial_{11}
$$

\section{III.Proposed Algorithm For CLG}

The unknown functions on a rectangular pixel grid of size $h$ are $u(x, y, t)$ and $v(x, y, t)$ and $u_{i}$ is the approximation to $u$ at some pixel $i$ with $i=1, \ldots . \mathrm{N}$. Gaussian convolution is realized in the spatial/spatiotemporal domain by discrete convolution with a truncated and renormalized Gaussian, where the truncation took place at 3 times the standard deviation. Symmetry and reparability has been exploited in order to speed up these discrete convolutions. Spatial derivatives of the image data have been approximated using a sixth-order approximation with the stencil $(-1,9$, $45,-9,1) /(60 \mathrm{~h})$. Temporal derivatives are either approximated with a simple two two-point stencil or the fifth-order approximation $(-9,125,-2250,2250,-125$, $9) /(1920 \mathrm{~h})$. If $J_{m n i}$ is the component $(n, m)$ of the structure tensor $J_{\rho}(\nabla f)$ in some pixel $i$ and $N(i)$ denotes the set of (4 in 2-D, 6 in 3-D) neighbors of pixel $i$, then a finite difference approximation to the Eular-Lagrange equations are given by Eqn. 16 and Eqn. 17. 


$$
\begin{aligned}
& 0=\sum_{j \in N(i)} \frac{u_{j}-u_{i}}{h^{2}}-\frac{1}{\alpha}\left(J_{11 i} u_{i}+J_{12 i} v_{i}+J_{13 i}\right. \\
& 0=\sum_{j \in N(i)} \frac{v_{j}-v_{i}}{h^{2}}-\frac{1}{\alpha}\left(J_{21 i} u_{i}+J_{22 i} v_{i}+J_{23 i}\right.
\end{aligned}
$$

Where $i=1, \ldots \mathrm{N}$. This sparse linear system of equations has been solved the Successive Over-Relaxation (SOR) method because of its simplicity and efficiency. If the upper index denotes the iteration step, the SOR method can be written as Eqn. 18 and Eqn. 19.

$$
\begin{gathered}
u_{i}^{k+1}=(1-\omega) u_{i}^{k} \\
+\omega \frac{\sum_{j \in N^{-}(i)} u_{j}^{k+1}+\sum_{j \in N^{+}(i)} u_{j}^{k}-\frac{h^{2}}{\alpha}\left(\mathrm{J}_{12 \mathrm{i}} v_{i}{ }^{k}+\mathrm{J}_{13 \mathrm{i}}\right)}{|N(i)|+\frac{h^{2}}{\alpha} \mathrm{J}_{11 \mathrm{i}}} \\
\omega \frac{\sum_{j \in N^{-}(i)} v_{j}^{k+1}+\sum_{j \in N^{+}(i)} v_{j}^{k}-\frac{h^{2}}{\alpha}\left(\mathrm{J}_{21 \mathrm{i}} u_{i}^{k+1}+\mathrm{J}_{23 \mathrm{i}}\right)}{|N(i)|+\frac{h^{2}}{\alpha} \mathrm{J}_{22 \mathrm{i}}}
\end{gathered}
$$

where

$$
N^{-}(i)=\{j \in N(i), j<i\}, N^{+}(i)=\{j \in N(i), j>i\}
$$

Here $|N(i)|$ denotes the number of neighbors of pixel $i$ that belongs to the image domain. The relaxation parameter $\omega \epsilon[0,2]$ has a strong influence on the convergence speed. For $\omega=1$ one obtains the wellknown Gausseidel method. Usually the used values for $\omega$ are between 0.9 and 1.99. This numerically inexpensive over relaxation step results in a speed-up by one order of magnitude compared with the Gauss-Seidel approach. The flow components for the first iteration are initialized by 0 . The specific choice of the initialization is not critical since the method is globally convergent. It should be noted that the iteration scheme does not require many computations per step, since one may compute expression of type $\left(\frac{h_{2}}{\alpha}\right) J_{n m i}$ before entering the iteration loop. Moreover any practical implementation requires only a single vector of size $\mathrm{N}$ for storing each of the two flow components $\mathrm{u}$ and v. Since the components are updated sequentially, there is no need for two vectors for the iteration levels $\mathrm{k}$ and $\mathrm{k}+1$. The algorithm is applied on a sequence of image with $\mathrm{T}$ frames in this paper.

\section{Algorithm 1 (Optical Flow Field Construction)}

Input: A sequence of images with $\mathrm{T}$ frames, iteration $\mathrm{k}$ for SOR, $\alpha, w$ and $\rho$.

Method: The Optical Flow Field is constructed in the following steps.

1. Read files in array of matrix

2. For every frame, $t$, perform the following steps:

i. Pre-smooth using the Gaussian Kernel with standard deviation $\alpha$. ii. Calculate the deviation of image $f_{x}, f_{y}, f_{t}$.

iii. Compute the velocity $\mathrm{u}, \mathrm{v}$ of image $\mathrm{t}$ using Successive Over-Relaxation (SOR) method for $\mathrm{k}$ iteration.

iv. Calculate the resultant velocity and angle and draw.

v. Save in file.

3. End.

\section{IV.EXPERIMENTAL RESULTS}

The effectiveness of the method has been justified over some experiments. All the applications and experiments have been done on MATLAB 7.9 R2009a and windows XP service pack 2 . The computers configuration was Intel Core 2Duo 2.2 GHz, 2 GB RAM. The proposed algorithm has been applied in the famous Yosemite Picture sequence, which is universally used for Optical flow checking. It is necessary to weak the parameters to get the correct result as the ground truth flow.

Here in this paper is presented the ground truth flow of the Yosemite picture 8th and 9th frame along with the Yosemite picture itself. In the Yosemite picture the proposed algorithm is approximately accurate except for the cloud region flow. The flow field density around the cloud region is a bi diverging and out of order comparing with the ground truth flow. There was other part where it was a little bi-off the track but that was all the matter of parameter optimization and also image differentiation approximation try outs. The main problem was to implement the optical flow algorithm in a manner where the differentiation approximation and parameters would coincide with each other to provide a better result. The parameters are actually application dependent i.e for different applications the parameters are also different. Here the proposed method has been applied on 32 sequences of medical images. Parameters value was also changed. For the Yosemite sequence, the used value for $\alpha, \sigma, \rho, \mathrm{h}$ and $\omega$ are $0.5,1.77,15,1$ and 1.9 respectively. For the medical image $\alpha=1, \sigma=0, \rho=5, h=1, \omega=1.99$ shows the best result among various combination of values we have tried. The 1st frame of Yosemite sequence is shown in Fig. 1. Fig. 2 shows the ground truth of Yosemite image sequence.

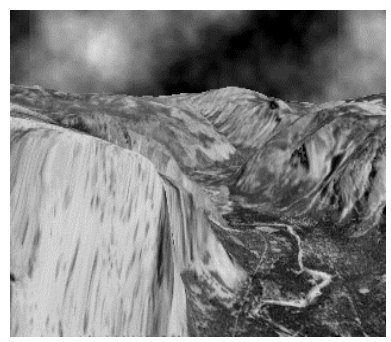

(a)

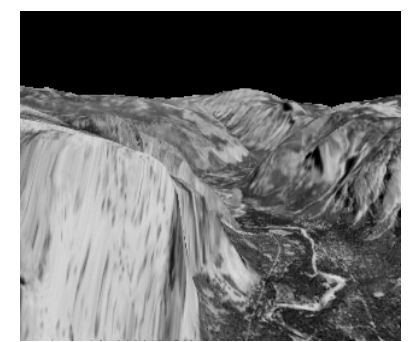

(b)
Fig 1: The Yosemite image sequence (a) Yosemite with clouds (b) Yosemite without clouds 
Vol. 4, Issue 2, February 2015

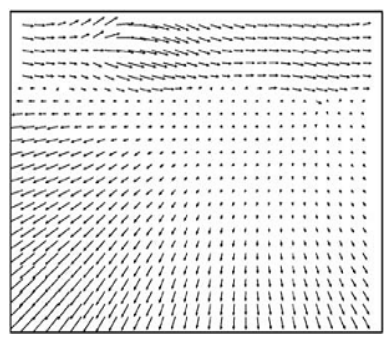

(a)

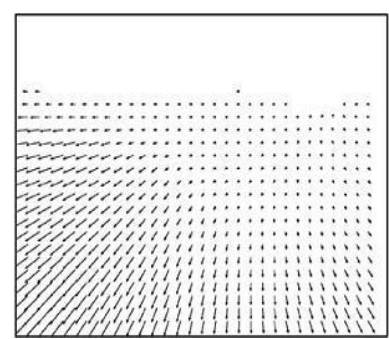

(b)
Fig 2: Ground truth flow field of the Yosemite image sequence (a) With clouds (b) Without clouds

As the comparison is made with Local (Lucas-Kanade) and Global (Horn-Schunk) optical flow method of motion detection. So the motion detected by Local and Global optical flow method is shown by Fig 4.

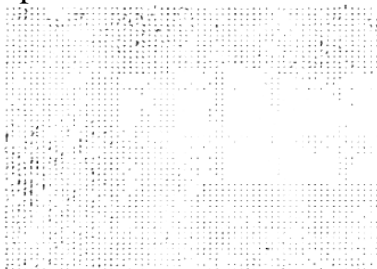

(a)

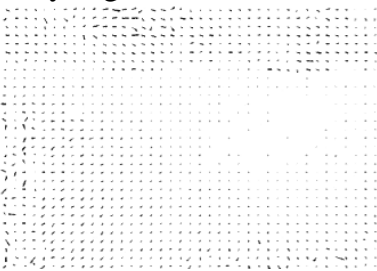

(b)
Fig 3: Optical flow of Yosemite with clouds using a) HomSchunk (Global Flow) method b) Lucas-Kanade (Local

The development algorithm has been tested in this Yosemite image and the found optical flow field is illustrated in Fig. 4.

As mentioned before that $\alpha$ is the regularization constant used to regularize the flow field. It has a significant role in optical flow determination. The results showing the effect

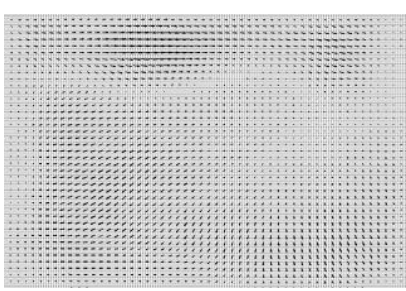

(a)

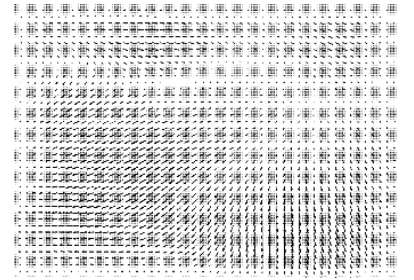

(b)
Fig 4: Optical Flow of Yosemite using developed algorithm a) With clouds b)Without clouds. Thevalue of parameters used are $\alpha=.07$, $\sigma=1.77, \rho=15, h=1, w=1.91$ (optimum valuedetermined)

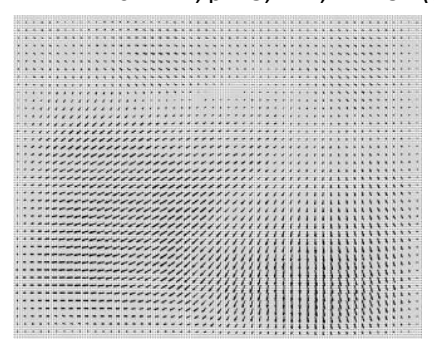

(a)

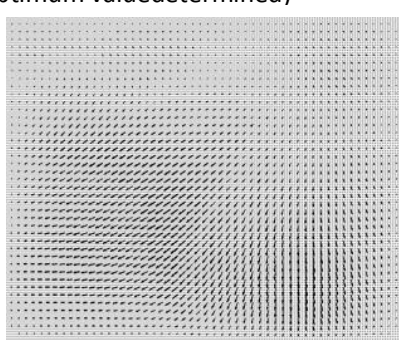

(b)
Fig 5: Optical flow field using Combine Local Global method a) using $\alpha=.001$, ite=100, $\rho=15, w=1.91, b)$ using $\alpha=.008$, ite $=100$,

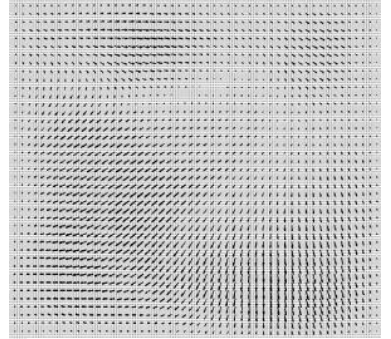

(a)

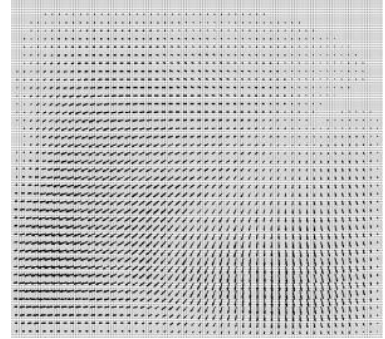

(b)
Fig 6: Optical flow field using Combine Local Global method

a) using $\alpha=.2$, ite $=100, \rho=15, w=1.91$ b) using $\alpha=.5$, ite $=100, \rho=15$, ot $\alpha$ in the flow tield are turnished in Fig. 5 and Fig. 6 , respectively.

From these figures it is obvious that too much regularization diminishes the top flow of the Clouds. That is each application may have the regularization parameters results best when $\alpha$ is from 0.05 to 0.09 for 100 to 1000 iteration. The pre-smoothing plays a significant role in the algorithm. Optimum values were thus found out by experimenting with the value of Standard Deviation of the Gaussian Kernel of Pre-smoothing constraint. Fig 7 shows the optical flow field using Hybrid Local Global method for various value of $\alpha$.

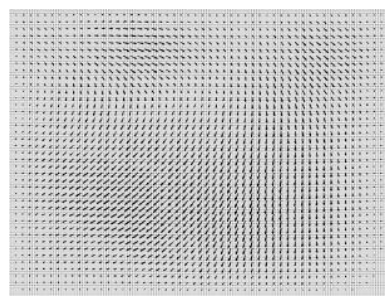

(a)

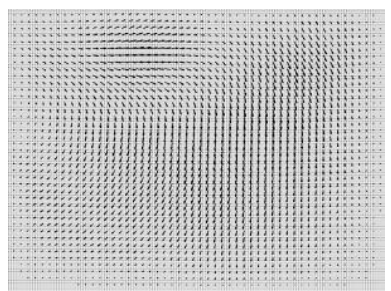

(b)
Fig 7: Optical flow field using Combine Local Global method

a) using $\alpha=.07, \rho=15, w=1.91, \sigma=5$ b) using $\alpha=.07, \rho=15$,

The parameter $\omega$ works as a converging constant. It fastens the converging process of the successive over relaxation iterative method.

It is seen from experience that it does not affect the optical flow as a whole. But higher iteration number changes the flow a little bit. Fig. 8 shows the optical flow field using Hybrid Local Global method for various values of $\alpha, \omega$ and $\sigma$.

It is clear from the figure that optical surely deteriorates because of too much smoothing. The optical flow spreads in the region where it should not be.

The pre-smoothing value was also tested with other values of $\alpha, \omega$. The result was same, optical for $\sigma=1.77$ to 1.95 . Another parameter which affects the optical flow is called integration scale of derivation, $\mathrm{K} \rho$ of standard deviation $\rho$. It helps the velocity constraints to produce smoother velocity flow. But greater values of $\rho$ tend to lessen the flow field. 


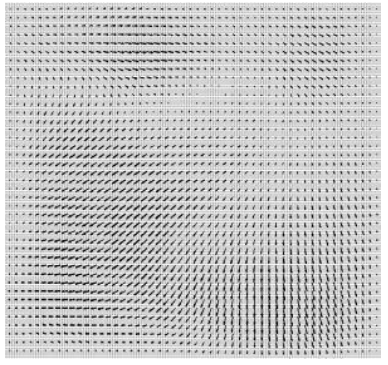

(a)

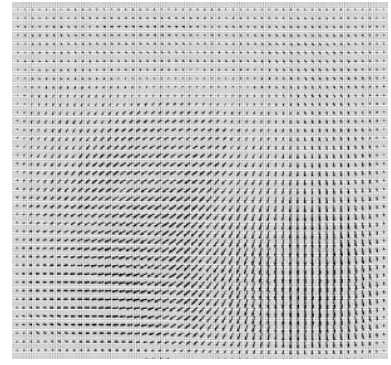

(b)

Fig 8: Optical flow field using Combine Local Global method

a) using $\alpha=.07, w=1.91, \sigma=1.77, \rho=16, b)$ using $\alpha=.07, w=1.91$, CLu nas been appired on $3 \angle$ meaical images wnicn snow motion detection of blood flow. Among them 6 frames been shown in Fig. 9
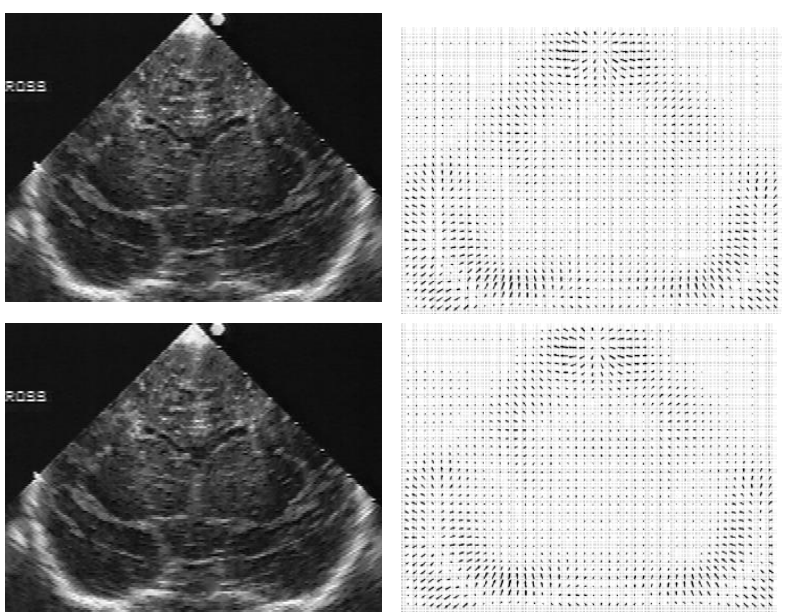

In this research Gaussian noise has been considered to compare the performance of different methods with the proposed method. Here the mean is 0 and the variance of Gaussian noise is changeable. The used parameter to compare the performance is Amplitude error which is represented in Eqn. 20

$$
\text { Amplitude Error }=\left|\frac{\|\hat{V}\|-|| V||}{|| V||}\right| * 100
$$
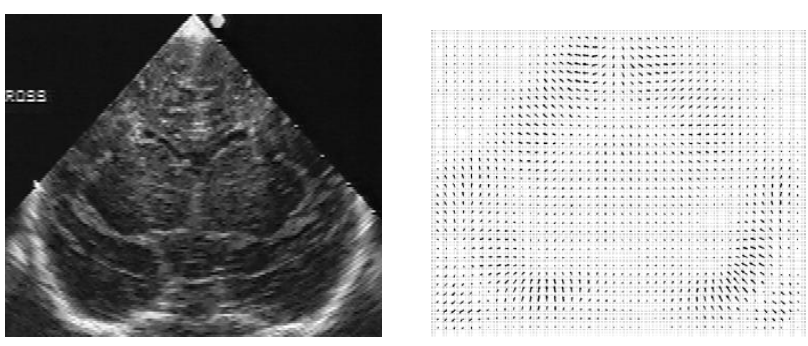

Where, $\widehat{V}$ and $V$ are the Estimated and Real vectors.

Table 1 and Fig. 10 reveal that the error for the CLG method is less than both the global and local method. So the Combined Local-Global Methods is less affected by noise can be extended to Bigun's structure tensor method into global energy function. Multi-resolution approach may also be found using the penalizers with a little mathematical work. 